\title{
Development of Optimal Accelerated Test Plan
}

\author{
Seyyedeh zohreh Fatemi, PhD student, University of Angers \\ Fabrice Guerin, PhD, University of Angers \\ Laurent Saintis, $\mathrm{PhD}$, University of Angers
}

Key Words: accelerated test, reliability, optimization, Bayesian estimation, testing cost, operation cost, robustness, Genetic algorithm.

\section{SUMMARY \& CONCLUSIONS}

This paper describes an optimal accelerated test plan considering an economic approach. We introduce a general framework to obtain plans of optimal accelerate tests with a specific objective, such as cost. The optimal test plans are defined by considering prior knowledge of reliability, including the reliability function and its scale and shape parameters, and the appropriate model to characterize the accelerated life.. This information is used in Bayesian inference to optimize the test plan. The prior knowledge contains the uncertainty on real reliability of new product. So, the proposed methodology consists of defining an optimal accelerated testing plan while considering an objective function based on economic value, using Bayesian inference for optimizing the test plan, and using the uncertainty of the parameters to obtain a robust, optimal testing plan. The objective function consists of two terms: the cost linked to testing activities and the cost associated with operation of the product.

Finally, we will develop our optimal plan by extending our approach to include theoretical formulation of the various degrees of freedom with respect to the parameters. To complete this development, we need to improve the algorithm of optimization. To obtain the best test plan, we propose an optimization procedure using the genetic algorithm. The proposed method will be illustrated by a numerical example based on a well-known problem.

\section{INTRODUCTION}

Accelerated Life Tests (ALTs) are widely used in reliability studies. Because many modern high-reliability components are expected to perform their proper functions for a very long time, simply testing these components under use conditions will usually yield little useful information about reliability within practical time and cost constraints. Accelerating variables, such as temperature, are often applied to obtain failures more rapidly. According to Figure 1, the resulting data at the higher stresses are used to estimate, through extrapolation with an appropriate acceleration model, the life distribution of the component at specified use conditions. Careful planning of an ALT is important to allow the most efficient use of limited resources, namely, time, number of test units, and the test facilities. Typically, ALT plans specify the levels of the accelerating variable and the quantity of available test units for these levels. With certain planning criteria, such as the estimation precision of a particular characteristic of the life distribution at use conditions, optimization can be used to find optimum test plan. Optimum test plans provide insight needed to obtain good practical test plans [1].

Accelerated Life Test (ALT) is a test method which subjects test units to higher than use stress levels in order to compress the time to failure of the units. Conducting a Quantitative Accelerated Life Test (QALT) requires the determination or development of an appropriate life-stress relationship model.

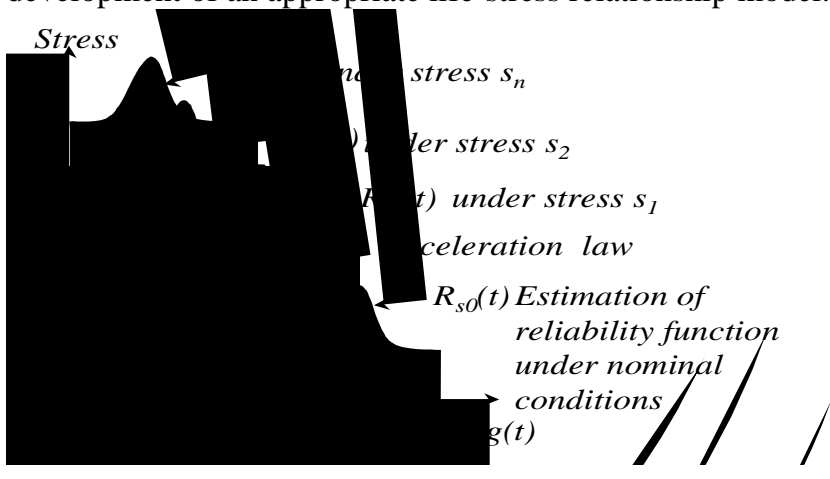

Figure 1- Principle of ALT test

Moreover, a test plan needs to be developed to obtain appropriate and sufficient information in order to accurately estimate reliability performance at operating conditions, significantly reduce test times and costs and achieve other objectives. One of the first decisions to be made when designing a reliability verification test is to determine how many units to test. If many units are tested, the duration of the test will be short. With this approach, prototype costs will be high, and development time costs will be low. If few units are tested, the duration of the test will be longer and prototype costs will be low, but development time costs will be high.

\subsection{Objective}

The objective of methodology is to define an optimal accelerated testing plan considering an economic approach. The objective function is defined by two terms: the cost linked 
to testing activities and the cost associated to operation of the product. In many works, the optimum testing plans are defined in considering a prior knowledge on reliability parameters. So, choice of reliability function and acceleration model and also having insight on the parameters such as scale and shape parameters is important. To evaluate the proportions of failure at each accelerated level. This prior knowledge is not integrated in statistical inference during the optimization process especially in minimization of reliability metric variance, and the estimation process with testing data. Nevertheless, this information can be used in Bayesian inference to optimize the testing plan, but it contains the uncertainty on real reliability of the new product. So, the proposed methodology consists of defining an optimal accelerated testing plan while considering an objective function based on economics, Bayesian inference for optimizing the test plan, and accounting for the uncertainty of parameters.

\subsection{Proposed method}

The proposed method is decomposed in several steps.

\section{Definition of reliability target}

The test plan is designed to demonstrate the contractual reliability metric target. Various metrics are used to characterize the reliability of products, such as MTTF, B10, or probability of failure for the warranty period. The verification consists of evaluating the risk of not reaching the reliability target using a point estimate and confidence interval.

\section{Prior knowledge on product}

The prior knowledge of a new product especially in the field Activation Energy and MTTF is obtained from Expert's opinion, Field data analysis on old product, Reliability Standard, etc.

\section{Test plan to optimize}

The kind of accelerated test plan is fixed at the beginning of study. We consider constant stress and number of stress levels and sample size are fixed. The decision variables of test plan optimization are chosen from among the test plan parameters.

\section{Objective Function}

The objective of the accelerated testing plan optimization is to minimize the global cost as defined by the costs of testing and operation. This term allows us to introduce a robustness analysis according through an objective function.

\section{Optimization procedure}

To obtain the best test plan, we propose an optimization procedure using the genetic algorithm. The proposed method will be illustrated by a numerical example.

\section{ESTIMATION IN PARAMETRIC ALT MODEL}

The parametric ALT model has been described in [1]. In this section, we assume the main results for a particular case of a constant stress, $S$, by the reliability function:

$$
R_{S}(t)=R_{S_{0}}(r(S) t)
$$

So, the stress affects only the scale parameter. Note that $r\left(S_{0}\right)=1$. If the function $r$ is completely unknown, the reliability function $R_{S_{0}}$ cannot be estimated. Therefore, the function $r$ is chosen as a particular class of functions. In survival analysis, log-linear models are frequently used as ALT as regression models.

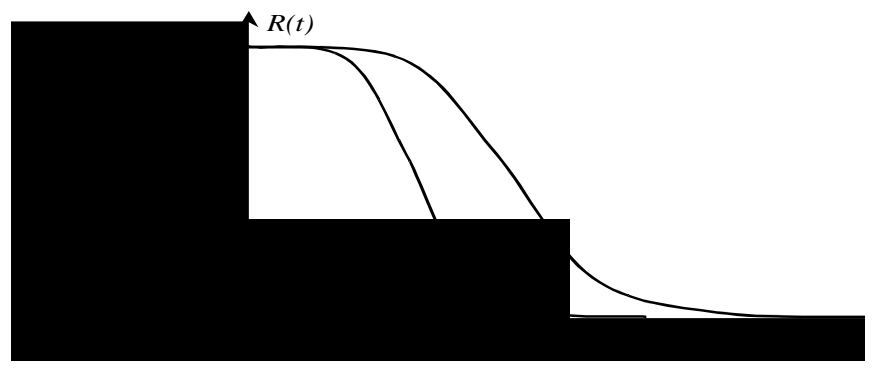

Figure 2- Definition of time transfer regression model $r(S)$

For the particular cases of constant stress, the equation (1) becomes (see Figure 2):

$$
R_{S}(t)=R_{S_{0}}\left(e^{\beta^{T} \cdot z} t\right)
$$

with

$$
\beta=\left(\beta_{0}, . ., \beta_{m}\right)^{T} \text { a } \quad \text { parameters }
$$

vector,

$z=\left(\varphi_{0}(S), . ., \varphi_{m}(S)\right)^{T}$ with the functions $\varphi_{i}$ specified and with the first component $z_{0}$ equal to 1 . Several models, as Arrhenius, inverse power, and the generalized Eyring, can be obtained as a particular case of this general form. It is assumed that the survival function $R(t)$ belongs to a class of functions depending only on the parameters of scale $\eta$ and shape $v$ [4]:

$$
R_{S_{0}}(t)=R_{0}\left(\left(\frac{t}{\eta}\right)^{v}\right),(\eta, v>0)
$$

Several models, such as Weibull and lognormal, are just particular cases of the above form $R_{0}(t)=e^{-t}, R_{0}(t)=1-\Phi(\ln t)$ respectively as detailed in [2].

The notations $R(u)=R_{0}\left(e^{u}\right), u \in \mathfrak{R}, u=\ln (t), \sigma=\frac{1}{v}$, $\gamma=\left(\gamma_{0}, \ldots, \gamma_{m}\right), \gamma_{0}=\ln \eta-\beta_{0}$ et $\gamma_{i}=-\beta_{i}, \mathrm{i}=(1, \ldots, \mathrm{m})$ allow us to rewrite the equation (2) as:

$$
R_{S}(t)=R\left(\frac{\ln t-\gamma^{T} Z}{\sigma}\right)
$$

The likelihood function can be written as:

$$
L(T \mid \gamma, \sigma)=\prod_{i=1}^{k} \prod_{j=1}^{n_{i}}\left\{\left[\frac{1}{\sigma} \lambda\left(\frac{T_{i j}-\gamma^{T} Z^{(i)}}{\sigma}\right)\right]^{\delta_{i j}} R\left(\frac{T_{i j}-\gamma^{T} Z^{(i)}}{\sigma}\right)\right\}
$$

Note: $T_{i j}$ is the life time observed or censored of the $j^{\text {th }}$ unit from $i^{\text {th }}$ stress level group.

\subsection{Point Estimate}

MLE chooses the parameters that maximize the likelihood 
of the data. In MLE, parameters are assumed to be unknown but fixed, and are estimated with some confidence. We consider that the failures are independent, meaning that the failure of one system does not affect another system.

If $L(\boldsymbol{T} / \theta)$ is differentiable and if the maximum likelihood $\hat{\theta}=\left(\hat{\theta}_{1}, \ldots, \hat{\theta}_{r}\right)$ exists, then it satisfies the following equation:

$$
\left.\frac{\partial L\left(t_{1} . . t_{n} \mid \theta_{1} . . \theta_{r}\right)}{\partial \theta_{j}}\right|_{\theta_{j}=\hat{\theta}_{j}}=0
$$

The computation of $R(t \mid \theta=\hat{\theta})$ allows us to approximate the reliability function associated with the product generating times of failure for the point estimate $\theta=\hat{\theta}$. An estimation of confidence intervals is required to complete the approach.

\subsection{Confidence Intervals}

The Cramer-Rao lower bound is a limit to the variance that can be attained by an unbiased estimator of a parameter $\theta$ of a distribution. Unbiased estimators enjoy a great popularity because they are easier to study than estimators that might have a lower MSE (Mean Squared Error), but that are biased. In particular, the MSE of an unbiased estimator is just its variance.

Let $\mathrm{X}(\mathrm{Y})$ be an estimator of any vector function of parameters, if $\mathrm{X}(\mathrm{Y})$ is an unbiased estimator of $\theta$, then the Cramer-Rao bound states:

$$
\operatorname{cov}_{\theta}(X(Y)) \geq I(\theta)^{-1}
$$

With $I(\theta)=E\left[\left.\left(\frac{\partial \log L(\mathbf{T} \mid \theta)}{\partial \theta}\right)^{2}\right|_{\theta=\hat{\theta}}\right]$ the Fisher information.

The confidence limits for the case of $\theta>0$ are obtained by processing the $\log \log (\theta)$ as a normally distributed variable:

$$
\hat{\theta} \times e^{-\frac{\frac{w_{1}-\alpha}{2} \sqrt{\operatorname{Var}(\hat{\theta})}}{\hat{\theta}}}<\theta<\hat{\theta} \times e^{\frac{\frac{w_{1-\alpha}}{2} \sqrt{\operatorname{Var}(\hat{\theta})}}{\hat{\theta}}}
$$

Moreover, for most of the cases, typical values for the parameters can be found. The values may be considered either as results of expertise using references as FIDES [3], as values associated with older/similar products, or as results of expert opinion(s).

\section{BAYESIAN INFERENCE}

The Bayesian approach to estimate the parameters is described in [1]. In this section, we present the formula used to obtain MAP means Maximum A Posteriori estimators that it is a mode of the posterior distribution. Bayesian inference uses a numerical estimate of the degree of confidence in a hypothesis before any evidence has been observed, and then it calculates a numerical estimate of the degree of confidence in the hypothesis after a set of evidence has been observed (This process is repeated whenever additional evidence is obtained).

The continuous form of Bayes theorem for the random variable $\theta$ over the $\Omega$ domain, having $t_{i}, i=1 . . n$ as test results, is:

$$
\pi_{\text {apo }}\left(\theta / t_{1}, . ., t_{n}\right)=\frac{L\left(t_{1}, . ., t_{n} / \theta\right) \pi(\theta)}{\int_{\Omega} L\left(t_{1}, . ., t_{n} / \theta\right) \pi(\theta) d \theta}
$$

with $\pi(\theta)$ the mathematical form, which formalizes the prior information.

The prior information over the normal conditions of use will be assimilated within the results of a number of tests already done hypothetically and added to the values of real tests. In Bayesian statistics, the uncertainty about the unknown parameters is quantified used probability so that the unknown parameters are regarded as random variables.

According to [1] the newly obtained $\pi_{\text {apo }}(\gamma, \sigma)$ function is a probability density function. So, by applying MLE theory and searching for values that maximizes $\pi_{a p o}(\gamma, \sigma)$, the variances associated to these estimators will be derived.

Differentiating the function $\ln \left[\pi_{\mathrm{apo}}(\gamma, \sigma)\right]$ with respect to $\gamma_{\mathrm{i}}$ and $\sigma$ results in the terms:

$$
U_{l}(\gamma, \sigma)=\frac{1}{\sigma} \sum_{i=1}^{k} z_{i l} \sum_{j=1}^{n_{i}} a_{i j}(\gamma, \sigma)+\frac{\mathbf{d} \ln \left[\pi_{\gamma_{l}}(\gamma, \sigma)\right]}{\mathbf{d} \gamma_{l}}
$$

With $1=1, \ldots, \mathrm{m}$ and:

$$
\begin{aligned}
& U_{m+1}(\gamma, \sigma)=\frac{1}{\sigma} \sum_{i=1}^{k} \sum_{j=1}^{n_{i}}\left\{v_{i j}(\gamma, \sigma) a_{i j}(\gamma, \sigma)-\delta_{i j}\right\}+\frac{\mathrm{d} \ln \left[\pi_{\sigma}(\gamma, \sigma)\right]}{\mathrm{d} \sigma} \\
& v_{i j}(\gamma, \sigma)=\frac{T_{i j}-\gamma^{T} Z^{(i)}}{\sigma} \\
& a_{i j}(\gamma, \sigma)=\lambda\left(v_{i j}(\gamma, \sigma)\right)-\delta_{i j}(\ln \lambda)^{\prime}\left(v_{i j}(\gamma, \sigma)\right) \\
& \frac{\partial \ln \left[\pi_{\sigma}(\gamma, \sigma)\right]}{\partial \sigma}=\frac{\mathrm{d}\left(\ln \left[d\left(\frac{\sigma}{c}\right)^{d}\right]+\ln \left[f_{0}^{\{m+1\}}\left[\left(\frac{\sigma}{c}\right)^{d}\right]\right]\right)}{\mathbf{d} \sigma} \\
& \frac{\partial \ln \left[\pi_{\gamma_{l}}\left(\gamma, \gamma_{l}\right)\right]}{\partial \gamma_{l}}=\frac{\mathbf{d}\left(\ln \left[b\left(\frac{\gamma_{l}}{a}\right)^{b}\right]+\ln \left[f_{0}^{\{l\}}\left[\left(\frac{\gamma_{l}}{a}\right)^{b}\right]\right]\right)}{\mathbf{d} \gamma_{l}}
\end{aligned}
$$

The MAP estimators $\hat{\sigma}, \hat{\gamma}$ can be obtained by solving the equation system:

$$
U_{p}(\gamma, \sigma)=0(p=1, \ldots, m+1)
$$

\section{OPTIMIZATION PROBLEM AND SIMULATION}

\subsection{Principle of proposed methodology}

The proposed methodology consists of defining an optimal accelerated testing plan while considering an objective function based on economic approach, using Bayesian inference for optimizing the test plan, and taking into account the uncertainty on parameters. This will produce a robust, optimal testing plan. To obtain the best test plan, we propose an optimization procedure using the genetic algorithm. The proposed method is decomposed in different steps as depicted in Figure 3.

In the next subsections, the different steps are detailed by considering a simple example to illustrate the methodology. 


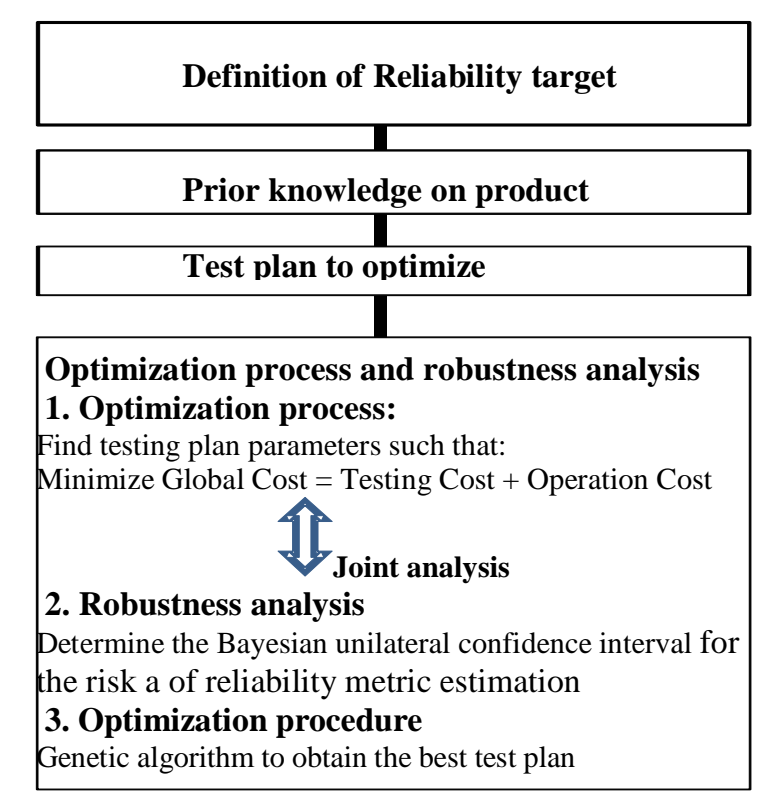

Figure 3-Principle of proposed methodology

\subsection{Definition of reliability target}

The test plan is designed to demonstrate the contractual reliability metric target. Various metrics are used to characterize the reliability of products, including MTTF, $B_{10}$, and probability of failure for the warranty period. The verification consists of evaluating the risk of failing to reach the reliability target in terms of the point estimate and confidence interval. In this paper, the probability of failure $p_{\text {operation }}$ for the operation time $t_{\text {operation }}$ is considered [6].

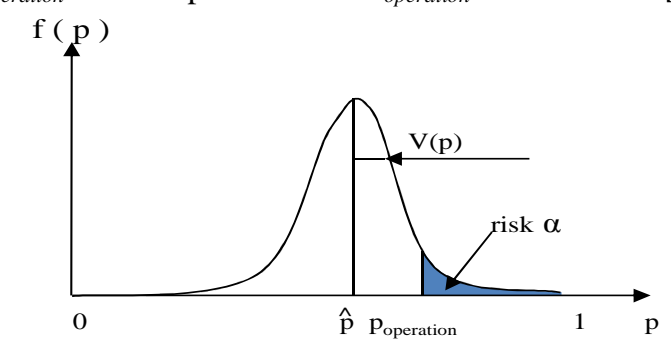

Figure 4- Probability function of operation failure $f(p)$

The point estimate is defined by

$$
\hat{p}=1-e^{-\left(t / e^{\left(\gamma_{0}+\gamma_{1} S\right)}\right)^{1 / \sigma}}
$$

and the variance of probability

$$
V(p)=\left(\frac{\partial p}{\partial \gamma_{0}}\right)^{2} V\left(\gamma_{0}\right)+\left(\frac{\partial p}{\partial \gamma 1}\right)^{2} V\left(\gamma_{1}\right)+\left(\frac{\partial p}{\partial \sigma}\right)^{2} V(\sigma)
$$

The probability of failure in operation period is estimated by the unilateral confidence interval for the risk $\alpha$ (see Figure 4). The beta distribution, $f(p)$, is used to characterize the distribution of $p$. The beta parameters are estimated by the moment's method:

$$
\begin{aligned}
& a_{0}=\frac{\hat{p}^{2}}{V(p)}(1-\hat{p})-\hat{p} \\
& b_{0}=\frac{\hat{p}}{V(p)}(1-\hat{p})^{2}+\hat{p}-1
\end{aligned}
$$

The probability of operation, $\mathrm{p}_{\text {operation }}$, is derived from:

$$
p_{\text {operation }}=\text { beta }^{-1}\left(1-\alpha \mid a_{0}, b_{0}\right)
$$

This probability represents the upper bound of the unilateral confidence interval of estimated probability of failure during the operation time.

The parameters $\hat{\gamma}_{1}, \hat{\gamma}_{0}, \hat{\sigma}, V\left(\hat{\gamma}_{1}\right), V\left(\hat{\gamma}_{0}\right), V(\hat{\sigma})$ are obtained by Monte Carlo simulation described by the following steps:

1. The generation of random values of $\gamma_{0}, \gamma_{1}$ and $\sigma$ from prior distribution $\pi_{\gamma 0}, \pi_{\gamma 1}$ and $\pi_{\sigma}$.

2. The generation of random time to failure with respect to censoring time $\tau$ at each stress level with random variables $\gamma_{0}$, $\gamma_{1}$ and $\sigma$ generated in step 1 .

3. The estimation of $\gamma_{0}, \gamma_{l}, \sigma, V\left(\gamma_{0}\right), V\left(\gamma_{l}\right)$ and $V(\sigma)$ by relationship (11).

4. The estimation of $p$ and $V(p)$ by relationships (12) and (13). 5. To repeat steps 1 to $4 n_{\text {simulation }}$ times. This repetition accounts for the sample size effect and the uncertainty on real reliability of new product characterized by the prior distributions.

6. To estimate the probability of operation in equation (14) with considering the means of $p$ and $V(p)$ on all repetitions.

\subsection{Prior knowledge on product}

By the assumption of independence between the variables, the joint distribution can be defined as:

$$
\pi\left(\gamma_{0}, \gamma_{1}, \sigma\right)=\pi_{\gamma_{0}}\left(\gamma_{0}\right) \times \pi_{\gamma_{1}}\left(\gamma_{1}\right) \times \pi_{\sigma}(\sigma)
$$

The choice of the form of $\pi$ depends on degree of knowledge on parameter $\left(\gamma_{0}, \gamma_{1}\right.$ or $\left.\sigma\right)$.

\subsection{Test plan to optimize}

In this paper, according to Figure 5, a simple test plan with three accelerated constant stress levels is studied by the following process:

- the censoring time is fixed

- $\quad$ the sample size $n$ is fixed

- the stress $S_{3}$ is fixed to maximum

- the middle stress level is unknown, because it depends on transformed stress factors

- the proportion allocation $p_{1}, p_{2}$ of sample size at stress level $S_{1}, S_{2}$ is unknown $\left(S_{2}\right.$ depend on $\left.S_{1}\right)$ and $\left(n_{1}=\right.$ $\operatorname{ent}\left(p_{1} . n\right) ; \mathrm{n}_{2}=\operatorname{ent}\left(p_{2} . n\right)$ and $\left.n_{3}=n-n_{1}-n_{2}\right)$

The decision variables of test plan optimization are:

- $\quad$ the middle and lower stress level $S_{1}$ and $S_{2}$

- the proportion allocation $p_{1}$ and $p_{2}$ of sample size respectively at stress level $S_{1}$ and $S_{2}$. 


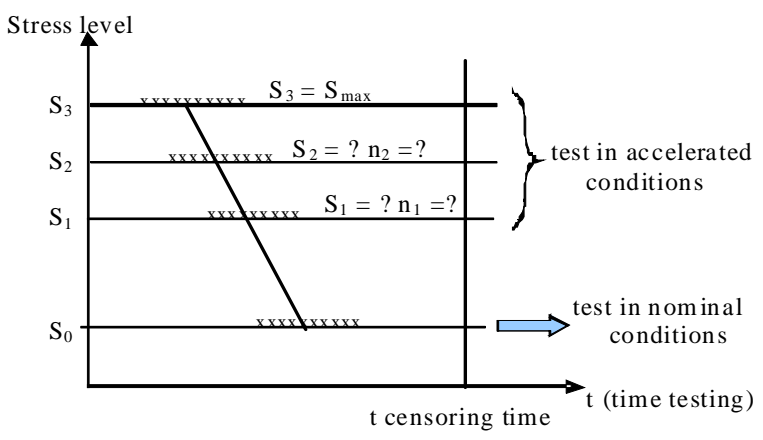

Figure 5-optimal test plan

\subsection{Objective Function}

The objective of the accelerated testing plan optimization is to minimize the global cost, which are defined by the costs of testing and operation, as expressed in [6]:

$$
\mathrm{C}_{\text {global }}=\mathrm{C}_{\text {testing }}+\mathrm{C}_{\text {operation }}
$$

where

$C_{\text {testing }}=$ fixed testing cost $+n \times$ unit price

$$
\begin{aligned}
& +\tau \times \text { cost per testing hour } \\
& +\left[\left(\frac{n_{1}}{N b}\right)^{\gamma_{\text {testing }}}+\left(\frac{n_{2}}{N b}\right)^{\gamma_{\text {testing }}}+\left(\frac{n_{3}}{N b}\right)^{\gamma_{\text {testing }}}\right] \\
& \quad \times \text { cost per batch }
\end{aligned}
$$

$\mathrm{Nb}$ represents the maximum number of units per batch.

$\gamma_{\text {testing }}$ represents the critical index for increasing the number of batch per stress level.

$C_{\text {operation }}=$ fixed operationcost

$$
+\left(p_{\text {operation }}-p_{\text {target }}\right) \times \text { product populationunit cost }
$$

+ cost of brandimageloss $\times\left(p_{\text {operation }}-p_{\text {target }}\right) \gamma_{\text {operation }}$

$p_{\text {target }}$ represents probability of failure for the warranty period.

$\gamma$ operation represents the critical index for brand image loss.

The operation cost is defined by considering risk $\alpha$ in terms of $p_{\text {operation. }}$ This term allows us to introduce a robustness analysis according to objective function.

With the test plan definition in $4.4, p_{\text {operation }}$ is function of $p_{1}, p_{2}, S_{1}$ and $S_{2}$, and the optimization model can be written as follows:

$$
\operatorname{Min}_{p_{1}, p_{2}, S_{1}, S_{2}}\left(C_{\text {global }}\right)
$$

Subject to $p_{1}, p_{2} \in[0,1], p_{1}+p_{2} \leq 1, S_{3} \leq S_{2} \leq S_{1} \leq S_{0}$.

\subsection{Optimization procedure}

To shorten the simulation time required, it can be assumed that the test plan will be correct for a given theoretical distribution of failures for each stress. It has been shown this method can lead to good test plans but it has not been demonstrated that it led to the best one. Thus, it is better to consider all the possible plans and to quickly converge to the best solution.

To obtain the best test plan, several optimization algorithms can be applied, including Least-mean-square and simulated annealing. In previous work [6], we used a response surface methodology to reach the optimum on a reliability ball bearing test plan. This methodology has provided good results but it was really dependent on the number of input parameters. In this project, we propose an optimization procedure using the Genetic Algorithm (GA). The reasons of this choice are that GA is a quite simple and efficient technique to be applied with heterogeneous inputs, especially in research of an optimum accelerated life test plan [5]. Moreover, GA allows us to search the optimum with more freedom inputs.

The GA has been firstly presented by J. Holland in 1975 [9]. The GA is a search and optimization technique based on the mechanism of evolution.

In GA, the individuals are generated randomly in order to construct a population. After estimating the fitness of individuals, parents are selected from the population according to the fitness value. Offspring are generated from the parents by using genetic operators such as the mutation or crossover.

Each element of the population is defined by a combination of $\left(p_{1}, p_{2}, S_{1}, S_{2}\right)$ with constrains explained in formula (17). With a discretization using integer values $\left(n_{1}\right.$, $n_{2}$ ) and temperatures, the formula (17) leads to the population's length: $N_{p o p}=2.3310^{6}$. For simulation of each test to 50 times, the fastest calculators need about 1 month of time. The fitness function corresponds to the global cost detailed in (16) and the programming is performed by GA MATLAB function.

\section{NUMERICAL EXAMPLE}

In this section, we will compare our results with the best compromise test plans for the Weibull distribution with one accelerating variable that Yang found on electronic module for pump control [7]. We also show the results of simple comparison studies between GAs and the response surface methodology.

\subsection{Yang's compromise test plans}

In this numerical example, the high stress must be specified. It should be as high as possible to yield more failures and decrease the variance of the estimate at the use stress. However, it should not cause failure modes that are different from those at the use stress. The low stress and the respective sample allocation are optimized by minimizing the variance of the estimate of the mean log life at use stress. The middle stress is equally spaced between the low and high levels and the corresponding number of test units is specified to be a half of that at the high stress [7]. Other test plan constraints may be found in, for example, Meeker [8].

Yang determined the best compromise test plan that minimizes the variance of the MLE of the mean log life at the use stress level [7]. For the smallest extreme value distribution, the mean equals the $43^{\text {rd }}$ percentile. The variance of the MLE of the mean, denoted $\hat{t}_{0.43}$, at the use stress level is given by 


$$
\operatorname{Var}\left[\hat{t}_{0.43}\right]=\frac{\sigma^{2}}{n} V
$$

where $V$ is called the standardized variance. The formulation of $V$ is given in [8].

\subsection{Simulation data}

We consider an electronic module for pump control that normally operates at $45^{\circ} \mathrm{C}$. To estimate its reliability at the use condition, 50 units are to be tested at three elevated temperatures. The high one is $105^{\circ} \mathrm{C}$, which is $5^{\circ} \mathrm{C}$ lower than the maximum allowable temperature. The censoring times are fixed for low, middle and high stress levels respectively at 1080, 600 and 380 hours.

\subsection{The Comparisons}

The objective is:

- Comparison with results obtained from the response surface of the experimental plan of Yang (A priori and different cost function).

- Comparison of the results obtained from the Genetic Algorithm in "releasing" a constraint on $\mathrm{n}_{2}$ (Comparison in computation time and optimum).

\subsection{The Results}

The table 1 gives the results by proposed approach (GAs) and Yang's test plan.

The results are identical and show that the GAs optimization generates the same results. In future work, we propose to liberate other parameters such as the censoring times and the number of stress levels.

\begin{tabular}{|c|c|c|c|c|}
\hline & Group & 1 & 2 & 3 \\
\hline \multirow[t]{2}{*}{ Yang } & $\begin{array}{l}\text { Number of } \\
\text { Test Units }\end{array}$ & 34 & 5 & 11 \\
\hline & $\begin{array}{l}\text { Temperature } \\
\left({ }^{\circ} \mathrm{C}\right)\end{array}$ & 74 & 89 & 105 \\
\hline \multirow{2}{*}{$\begin{array}{l}\text { GA (with } \\
\text { same } \\
\text { constraints) }\end{array}$} & $\begin{array}{l}\text { Number of } \\
\text { Test Units }\end{array}$ & 34 & 5 & 11 \\
\hline & $\begin{array}{l}\text { Temperature } \\
\left({ }^{\circ} \mathrm{C}\right)\end{array}$ & 74.54 & 88.98 & 105 \\
\hline
\end{tabular}

Table 1- The comparison of GA and Yang's results

- According to table 1, you can see that the GA technique and Yang's results have a close relationship.

- MTTF; to identify the probability of overlapping our findings and Yang's results.

\section{CONCLUSION}

In this paper, we introduced a general framework to obtain optimal accelerate test plans with a cost objective. The cost objective function is developed in a theoretical formulation with the test plan parameters. Then, this new framework is compared with the results obtained from Genetic algorithm.
A genetic algorithm is often a discrete stochastic process that can be considered as a Markovian process. It means that several results can derive from the theory of this kind of process, which enables one to easily verify the optimization's efficiency [5]. Moreover, GA is a simple and efficient method, so in the future we can use this technique as a good alternative method for estimation of probabilities.

\section{REFERENCES}

1. S. Voiculescu, F. Guérin and M. Barreau. "Bayesian parameter estimation with prior weighting in ALT model," Proc. Ann. Reliability \& Maintainability Symp., (Jan.) 2009, pp 53-58.

2. L. C. V. Nikulin, M, Gerville-Réache and V Couallier, Statistique des essais accélérés, Hermes Science, 2007.

3. FIDES, FIDES Guide 2004: "Reliability Methodology for Electronic Defense System", 2004.

4. V. Bagdonavicius, M. Nikoulin, "Transfer functional and semi parametric regression models", Boimetrika, vol. 84(2), 1997, pp 365-378.

5. P. Lantieri, F. Guérin. "Optimization of a Step-stress accelerated life test plan by genetic algorithm", Quality assurance, Romanian Society Quality Assurance (SRAC), Vol. 58, (April.) 2009.

6. S. Z. Fatemi, F. Guérin, L. Saintis. "Optimal accelerated test plan: economic approach”, ISSAT conference 2011.

7. G. Yang. Life Cycle Reliability Engineering, NJ, USA, John Wiley \& Sons, Inc., Hoboken.

8. W. Nelson, W. Q. Meeker. "Theory for Optimum Accelerated Censored Life Tests for Weibull and Extreme Value Distributions", Technometrics, Vol. 20(2), 1978, pp 171-177.

9. J. Holland. "Adaptation in Natural and Artificial Systems", Ann Arbor, University of Michigan Press, 1975.

\section{BIOGRAPHIES}

Seyyedeh Zohreh Fatemi, PhD Student

LASQUO Laboratory, ISTIA

62, Av. Notre Dame du Lac

Angers, Pays de la Loire, 49000, France

e-mail: szfatemi@rocketmail.com

Seyyedeh Zohreh Fatemi is $\mathrm{PhD}$ student in Reliability Engineering at University of Angers (France).

Fabrice Guérin, PhD

e-mail: fabrice.guerin@ univ-angers.fr

Fabrice GUERIN is a Professor at University of Angers.

Laurent Saintis, PhD.

e-mail: laurent.saintis@univ-angers.fr

Laurent Saintis is an associate professor at University of Angers (France). 